\title{
Conducting operational research during outbreaks to improve preparedness and response
}

\author{
Jennifer Nuzzo*, Matthew P. Shearer and Diane Meyer
}

Johns Hopkins Center for Health Security, Baltimore, MD, USA

\section{Objective}

The Outbreak Observatory $(\mathrm{OO})$ aims to:

- Strengthen outbreak/epidemic preparedness and response activities through real-time, one-the-ground observations and analyses

-Identify best practices based on operational experience that are broadly applicable across outbreak response agencies

- Serve as an independent voice to advocate for policies that support preparedness and response activities based on expert assessment of the resources required to build and maintain necessary outbreak response capabilities

-Support local practitioners' efforts to publish their experiences

Sharing the firsthand experience of responders is critical for building outbreak preparedness and response capacity, and OO will serve as a dedicated mechanism to collect, analyze and disseminate this information

\section{Introduction}

Each significant outbreak and epidemic raises questions that must be answered in order to better inform future preparedness and response efforts, such as:

- What are the systems and resources needed to characterize an outbreak?

-What systems and resources are needed to bring an outbreak to a close?

While we can anticipate these types of questions, the absence of dedicated mechanisms to record operational experiences and challenges can result in valuable, ephemeral data that are crucial for improving outbreak response not being consistently collected or analyzed.

Participation in outbreaks by external experts can be instrumental in ensuring that this important operational information is documented, analyzed and shared with the broader public health community. There is a particular need for observers external to the response who can capture and analyze applied data about the operational response to outbreaks - eg, the systems and strategies involved in responding to the such events - in order to improve our understanding of best practices for detecting and responding to these events. These can then be shared so that the entire public health community can access and incorporate lessons learned into their own preparedness and response plans. External observers can also help describe the important work performed by local responders during outbreaks and advocate for necessary preparedness and response program resources.

The Outbreak Observatory is currently in a pilot phase and is looking for international and US partners who may be interested in collaborating with members of our team during their next outbreak response.

\section{Methods}

When an outbreak occurs, OO will reach out to our partners to assess their interest in having project team member(s) travel to their location to observe the ongoing outbreak for the purpose of collaborating on a joint analysis of the response. The team member(s) would engage with local officials to identify operational challenges and best practices to better understand their perspectives and experiences.
Prior to the OO team's arrival, they will provide local responders a list of sample questions that the team is interested in exploring for the purpose of potential future written analysis, with the goal of focusing on those questions that are most relevant to both the local and broader public health communities. Once a preliminary list of study questions is developed, team members will engage with local responders to discuss their experiences.

Once on location, the OO team member(s) will regularly report their findings back to the Project Director. The OO team will work with the Project Director and local partners to compose and submit the findings to a peer-reviewed journal, ensuring that local practitioners receive appropriate authorship credit.

\section{Results}

OO aims to fill gaps in existing health security literature by sharing the experiences of practitioners involved in outbreak responses and co-authoring peer-reviewed publications with those responders. We envision that these publications will be available more quickly than existing outbreak reports. We will disseminate our findings to pertinent policymakers, members of the broader biosecurity and public health communities and the public to ensure that important lessons reach all appropriate audiences, especially those responsible for planning and resource allocation decisions for outbreak and epidemic response. In support of this, we have created a communication platform (www. outbreakobservatory.org) to publish interim observations via rapid communication channels (eg, communications with policymakers, social media, blog posts, video logs). All publications will be developed in partnership with local practitioners.

\section{Conclusions}

The lessons learned from previous epidemic and outbreak responses are critical to informing future response efforts. However, this data is often lost in the midst of an outbreak, when responders are too busy with the situation at hand to collect and analyze operational data. Outbreak Observatory endeavors to bridge this research gap, helping to capture and analyze this data and making it available to the broader public health community.

\section{Keywords}

Preparedness; Outbreak; epidemic; Response

\section{Acknowledgments}

Dr. Carlos Castillo-Salgado, Athalia Christie and Dr. Noreen Hynes for their help in developing $\mathrm{OO}$.

*Jennifer Nuzzo

E-mail: jnuzzo1@jhu.edu 